\title{
Tensiones y retos sobre la mujer rural y su participación en la construcción de paz en Colombia
}

\section{Ana Marcela Bueno}

Licenciada en Trabajadora Social

(Universidad Colegio Mayor de

Cudinamarca)

Magíster en Investigación Social

Interdisciplinaria (Universidad Distrital

Francisco José de Caldas, Colombia)

Doctoranda en Trabajo Social (UNR)

Docente-investigadora (Universidad de La

Salle, Bogotá, Colombia)

Integrante del Grupo de investigación

Trabajo Social, Equidad y Justicia Social (CEDT)

Correo: abueno@unisalle.edu.co
Karin Viviana Suarez Puentes

Licenciada en Psicología (Corporación Universidad Piloto de Colombia)

Especialista en Docencia Universitaria

(Corporación Universidad Piloto de

Colombia)

Magíster en Psicología Jurídica

(Universidad Santo Tomas)

Integrante del Grupo de investigación

Trabajo Social, Equidad y Justicia Social (CEDT)

Correo:kvsuarez@unisalle.edu.co 
Resumen

La mujer rural y sus dinámicas de participación han sido temas abordados en el marco de los estudios de las ciencias sociales desde los años 90, época en la cual la política pública visibilizó la relevancia de esta población en la producción de la tierra. Sin embargo, el reconocimiento del papel que desempeñan en la sociedad a través de los diferentes ámbitos ha sido subvalorado. Hoy en Colombia, con la firma del acuerdo de paz, reconocer el rol que puede cumplir la mujer en el territorio rural tiene sentido por la relevancia que cobra su aporte como reproductora de una sociedad que renace, aprovechando sus experiencias en el ámbito de la organización y la participación social. Con el fin de comenzar a construir un marco de referencia sobre esta temática, se realizó una revisión de investigaciones nacionales e internacionales publicadas desde el año 20061 buscando dar cuenta de los estudios realizados y el estado del conocimiento sobre la participación de las mujeres rurales en la construcción de paz en Colombia.

Palabras claves

Mujer rural - Participación - Paz
Abstract

The rural woman and their dynamics of participation, have been topics tackled in the frame of the studies of the social sciences from the 90 s, period in which the public policies showed the relevancy of this population in the production of land. Nevertheless, the recognition of the role that they redeem in the society across the different settings has been undervalued. Today in Colombia, with the signature of the peace agreement, to admit the roll that the woman can fulfill in the rural territory makes sense due to the relevancy of their contribution as reproducer of a society which is reborn, making use of their experiences in the organization and social participation settings. To begin to construct a frame of reference on this subject, we realized a review of national and international investigations published from the year 2006 taking into consideration de existing studies and the state of the knowledge regarding the participation of the rural women in the construction of peace in Colombia.

Keywords

Rural woman - Participation - Peace

1 Pese a la decisión de incluir los documentos escritos desde 2006, se incluyeron algunos de años anteriores que por su relevancia. 
Tensiones y retos sobre la mujer rural y

\section{Introducción}

La realidad de las mujeres rurales en Colombia hoy es un asunto relevante para el feminismo latinoamericano en tanto se trata de un momento histórico en el que se está transitando de dinámica social de guerra hacia la construcción de paz, y en dicho camino se ha convocado a las mujeres como actoras claves en los contextos privados que, a su vez, contribuyen a las transformaciones de la sociedad, por ello sus aportaciones a lo público. En ese escenario, la localidad de Sumapaz, un territorio anexo al distrito capital, ha estado históricamente resistiendo los avatares que le ha planteado la guerra y en esa misma lucha las mujeres han jugado un papel muy importante que vale la pena visibilizar para dar cuenta de estas experiencias, que aportan de manera significativa a los diversos planteamientos que se vienen reconociendo hoy, para evidenciar que existen bases para continuar construyendo condiciones para una paz estable y duradera. En este escenario, el presente escrito pretende dar cuenta de algunos avances en diversos contextos del territorio colombiano desde propuestas feministas que reconocen estas diferencias.

La localidad de Sumapaz es la número 20 de la ciudad de Bogotá, considerada como la única puramente rural del Distrito Capital. En los años 70 se crea el Parque Nacional Natural Sumapaz, que tiene una extensión de más de 150.000 hectáreas y cubre los departamentos de Cundinamarca, Huila y Meta, sobre la cordillera oriental; el Páramo de Sumapaz presenta en su mayor parte características montañosas con diversas lagunas y zonas aún inexploradas, convirtiéndolo en uno de los más ricos en especies de flora colombiana (Parques Naturales de Colombia, 2017).

Sumapaz está dividido en tres corregimientos: San Juan, Nazareth y Betania, con sus respectivas veredas, habitadas por campesinos, quienes se sostienen a través de actividades económicas como el cultivo de papa y la ganadería. Asimismo, la historia del páramo más grande del mundo se enmarca en las diferentes épocas de conflicto que ha vivido Colombia; es así como desde la segunda mitad del siglo XIX con la colonización de la región de Sumapaz, se abrió paso a la 
aparición de escenario de distintos conflictos entre colonos y arrendatarios; y años después se agudizó a través de la violencia bipartidista. A pesar de lo anterior, los campesinos sumapaceños y sumapaceñas siempre han persistido en no perder sus tierras, generando una lucha constante por sus territorios, heredados por sus antepasados, evidenciando no sólo arraigo, sino capacidad de agremiación y participación desde la mirada rural.

El sistema patriarcal a lo largo de la historia determinó que hombres y mujeres configuraran un orden social en el cual cada uno ocupaba un lugar para responder a las exigencias de la cotidianidad desde lo privado y desde lo público, lo que en esencia establece la asignación de unos roles específicos de acuerdo con el sexo, que se han naturalizado en la división del trabajo. Pese a esta situación, en el ámbito rural tal división no es tan clara, pues el trabajo productivo también ha requerido del aporte de las mujeres para alcanzar las condiciones necesarias de supervivencia, aun sin ser reconocido ni remunerado. Adicional a ello, la inequidad de género plantea complicaciones para el acceso a la tierra por parte de las mujeres, situación que ha hecho que esta población se vincule con procesos de organización y participación que la empodera para la exigibilidad de sus derechos.

Es así como en el reconocimiento de la participación de las mujeres por el acceso a la tierra se identifica una serie de problemáticas recurrentes, sobre las que vale la pena detenerse e identificar puntos de avance y elementos no resueltos para la superación de las mismas (Deere, Lastarria-Cornhiel, y Ranaboldo, 2011). En experiencias colombianas, como es el caso de un grupo de mujeres campesinas en Antioquía, se analizaron los efectos que generan el acceso y control de la tierra por su parte, identificando que la propiedad está mayoritariamente en manos de los hombres, obligándolas a depender en términos de ejecución de iniciativas económicas. De igual manera, se evidenció la invisibilización de las mujeres en al ámbito productivo, asumiendo que tal labor es parte de las funciones que se le asignan en el marco de su rol reproductivo. Acompañado lo anterior a la subvaloración del trabajo en el marco de los sistemas ecológicos, dado que cuando son explotados por las mujeres, y en sus parcelas, considera- 
das pequeñas para un enfoque de desarrollo territorial, no son apreciadas por no generar recursos en el corto plazo, pues se les ubica en una lógica doméstica (Zuluaga, 2011); ello necesariamente subordina no sólo su condición de mujer, sino como tal de productora, dejándola por fuera del mercado en lo que se refiere al acceso a recursos.

Por otro lado, en Colombia es innegable la relación del conflicto armado con las dificultades de acceder a la tierra, pues justamente parte de las complejidades del conflicto se dan por el control de la misma. Este fenómeno es referido por Pinto (2011), quien expresa que efectos del conflicto son los altos índices de desplazamiento y desarraigo, eventos que afectaron históricamente a las mujeres en territorios como Córdoba, Sucre y los Montes de María, pues el control de ese territorio tuvo enfrentados a varios actores armados en diferentes momentos. Como respuesta a lo anterior, se gestaron organizaciones de mujeres que abogan por la búsqueda de estrategias que permitan superar las complejidades que les deja el conflicto y buscar nuevas apuestas en términos de la exigencia de derechos relacionados con la tierra y el territorio, en los que irónicamente la lucha no era solamente por reclamarles a los actores que las despojaron, sino por la igualdad de condiciones respecto a sus compañeros, pues se sostiene la constante de dar un lugar de inferioridad a las mujeres en la legislación y en las acciones de la reforma agraria.

En esta misma línea, Parrado e Isidro (2014) problematizan el caso de la participación política de las mujeres misak ${ }^{1}$, quienes forman parte de las iniciativas pro defensa del territorio, siendo víctimas de múltiples violencias en el ámbito privado y en los cabildos y organizaciones indígenas. Es claro que su indignación se sustenta desde las diferentes prácticas de género de la comunidad indígena, en la que se identificó que la participación y liderazgo femenino debía estar acompañado de un liderazgo masculino que legitimara sus pedidos frente a la comunidad, evidenciando que el trabajo de la mujer frente al hombre no se encontraba en igualdad (Parrado e Isidro, 2014).

1 También conocidas como Guambianas: Este pueblo se localiza al noroeste del departamento del Cauca, en donde se concentran sus territorios ancestrales. 
La participación de las mujeres rurales es un proceso que a lo largo de la historia se ha ido configurando en la construcción social de los territorios en los que se ve cada vez más la necesidad de que ellas, como parte determinante del desarrollo rural, se vinculen en apuestas que abogan por su bienestar, el de sus familias y también el de sus comunidades. En dicho proceso, las mujeres se han visto enfrentadas a diversas situaciones que han complejizado su accionar, pero que a la vez les han motivado esfuerzos por posicionarse como actoras del cambio. Estos asuntos han sido referidos en investigaciones a partir de tres tópicos: las condiciones de la participación para las mujeres rurales (Arenas y Collazos, 2010; Sánchez y Moran, 2014; Perilla, 2014; y Villarreal, 2011); el empoderamiento de las mujeres a partir de la participación (Espinosa, 2012; Villarreal, 2011 y 2014; Villarreal y Ríos, 2006; Buendía-Martínez, 2013; y Espinosa y Olaya, 2012); y el papel del Estado las políticas públicas en los procesos de participación de las mujeres rurales (Rosado, 2011; y Castro y Molinari, 2014).

Es así que, en el estudio de dinámicas de la participación, el reconocimiento de cualquier territorio permite hacerse una idea de situaciones similares en otras sociedades rurales. Al respecto, Perilla (2014) analizó la dinámica específica de un territorio a partir del reconocimiento de una experiencia de encuentro con hombres y mujeres agricultores de papa en Nariño, sur del país. En ella, el problema central se refiere al triple rol que desempeña la mujer a nivel de lo productivo, reproductivo y comunitario, pero más allá de eso, cómo tal trabajo no es valorado significativamente, ni siquiera por ella misma. En esta experiencia ubicada en la seguridad alimentaria y la nutrición de la población, el asunto recae sobre las mujeres por ser quienes se encargan del cuidado. Con ello se evidencia, como en otras sociedades rurales, la clara clasificación tradicional de los roles de género que deben asumirse desde un triple rol independiente de las actividades que se desarrollen en el ámbito público. Adicional a ello, y como lo refirió Pinto previamente, el conflicto armado es otra constante que caracteriza a la mayoría de los territorios rurales, lo cual ha generado 
a lo largo de la historia procesos complejos en lo que se refiere a la organización y la participación.

Finalmente, en esta discusión también aportaron Espinosa y Olaya (2012), a partir del reconocimiento de una experiencia de organización en el municipio de Soracá, departamento de Boyacá, mostrando a partir del caso el interés que las comunidades rurales han manifestado por rescatar y revalorizar los cultivos ancestrales, evidenciando que la mujer rural ha venido asumiendo diversas actividades agropecuarias en el ámbito productivo, lo que ha llevado a que emprenda la vinculación con espacios organizativos que contribuyen a satisfacer necesidades e intereses personales y familiares - tales como la estabilidad económica a través del mejoramiento de la producción-, pero generan espacios democráticos que han surgido por necesidades e intereses personales que les permite fortalecer sus posibilidades de participación. Pese a la lucha por la ganancia de estos espacios hay insatisfacción por parte de los cónyuges, en tanto se considera que con este tipo de prácticas se subvierten los roles de género que a lo largo de la historia se han impuesto como inamovibles de acuerdo con la cultura patriarcal que subyace en todos los ámbitos de la sociedad, especialmente en el rural.

Por otro lado, se encuentra como aspecto fundamental la relación de las dinámicas de participación con el Estado. Es interesante el reconocimiento de diferencias tan marcadas en territorios muy cercanos separados por la frontera presentada por Rosado (2011). En estos casos, se debe tener en cuenta el compromiso que tenga cada Estado en las condiciones territoriales rurales. Este es un caso analizado en la frontera Colombo-venezolana en el que se identifica la incidencia que tienen los procesos políticos nacionales a cada lado de la frontera sobre las iniciativas organizativas de las mujeres indígenas Piaroa que habitan el curso medio del río Orinoco. En el proceso se visualiza cómo las políticas públicas de los dos países han llevado a estrategias diferentes de reivindicación y construcción de una nueva identidad cultural, que atraviesa por un ejercicio de pensar su posición como mujeres, indígenas y ciudadanas. A la vez que se reconoce que los impactos de los procesos políticos nacionales sobre el día a día de 
los habitantes de la frontera son una realidad, que implican cambios en los patrones culturales y establecen nuevas dinámicas de interacción con el otro. Se evidencia que parte de la necesidad creciente de las organizaciones de mujeres Piaroa por desarrollar las habilidades del mundo no indígena, se relaciona con las posibilidades de integración a la sociedad mayoritaria que como ciudadanas les otorgan las políticas impulsadas por los estados nacionales a ambos lados de la frontera.

Las investigaciones sobre construcción de paz desde las mujeres en Colombia son escasas, aún más en la especificidad de mujeres campesinas, aunque muchas de las experiencias son protagonizadas en este contexto. De manera crítica se puede evidenciar que no existen investigaciones sobre el tema en el Sumapaz y que más bien abundan los estudios ambientales y sobre ordenamiento territorial que tocan la región de manera parcial o total; existen algunos análisis sobre la dinámica del conflicto y organización campesina, pero ninguno se realiza desde el enfoque de género.

Frente al desarrollo teórico de mujer rural, se da cuenta de lo que es sociedad rural retomada desde Jordan (1989), quien la entiende como el espacio geográfico natural y de cultivo y en donde se desarrollan distintas actividades económicas como agricultura, ganadería, agroindustria, pesca, artesanías, comercio y servicios prestados por organismos privados y el Estado. Convergen grupos vinculados a la pequeña producción, trabajadores y trabajadoras asalariados, trabajadores del comercio, trabajadores de la industria y los servicios y las formas campesinas son aquellos sistemas de producción y consumo en los cuales la mano de obra familiar constituye el elemento organizativo de la producción y las necesidades de trabajo se satisfacen fundamentalmente sin contratación externa.

A la luz de las transformaciones que ha presentado el escenario rural han sido visibles los cambios en términos de organización económica, las relaciones de la población y la reproducción, lo que algunos comprenden como una nueva ruralidad en la cual se identifica la diversidad cultural que construye un entramado socioeconómico, la concepción de territorio ya sea por intereses económicos como de 
preservación ambiental, los estrechos vínculos con las esferas rurales y urbanas en el intercambio de mercancías, y el mercado. Aquí las mujeres juegan un papel fundamental en tanto ejercen múltiples actividades para sostener la economía y mantener el núcleo familiar; asumen las labores domésticas, su participación en los cultivos y el comercio. Adicional a ello, estos cambios han transformado los roles del hombre y de la mujer, asignando otras ocupaciones que permiten preservar la convivencia de la población.

En este marco, siguiendo a Deere y León (2000), se comprende que la tierra es fundamental para la reivindicación de los derechos de las mujeres ya que les permite tener independencia económica, vínculo para la negociación familiar, autonomía en las decisiones, y parte de la desigualdad radica justamente en los obstáculos que tienen las mujeres para acceder a la tierra que, por un lado, se asocia a las condiciones jurídicas que privilegian la titulación para los hombres y porque la apropiación de los territorios, ya sea a nivel individual o en procesos colectivos, es asumida por los hombres por considerarlos siempre los jefes de familia y, por ende, quienes toman las decisiones sobre ellas. Esto es reforzado con los programas institucionales que están pensados más en función de los varones, lo cual los hace discriminatorios y excluyentes. Sumado a ello, la propiedad de la tierra se concibe desde una mirada garantista de derechos, dado que es el escenario donde se pueden satisfacer los derechos como vivienda, alimentación, salud, educación vinculados a labores asignadas a las mujeres. Por ello, se reconocen como trabajo no remunerado. En este mismo marco, Lagarde (2000) refiere que los escasos recursos de las mujeres rurales se asocian con las relaciones de poder que subordinan el género femenino, ya que no se le brindan oportunidades y recursos que permitan solventar sus necesidades.

Para comprender los desarrollos que articulan el papel de la mujer rural en las dinámicas que en este ámbito se presentan es esencial articularlos con el concepto de género, categoría analítica nacida en los años 70 para dar cuenta de las desigualdades entre hombres y mujeres a partir del sistema patriarcal instalado en las sociedades a través de la historia; continuidad, además, de la lucha iniciada por las femi- 
nistas de la primera ola en el siglo XVII y XVIII en el período de la Ilustración, según lo contempla Cobo (1995). Los aportes de Poulain de la Barre fueron incidentes en el cuestionamiento de la teoría que sumerge a la mujer en una condición de inferioridad, por ello se reconoce que desde la época de la Ilustración se empieza a polemizar la consideración natural de la mujer y con ello la necesaria división de los espacios para la asignación natural de las tareas, seguido por la lucha que se dio durante el siglo XIX por alcanzar el derecho al sufragio y se complementó con las feministas de mediados de siglo XX que aportaron la categoría de género para problematizar la desigualdad. En esta línea se reconocen algunos aportes de autores como Lambert, D’Alembert, Méricourt, Gouges y Rousseau.

Para Latinoamérica fueron clave los planteamientos de Simone de Beauvoir que desata las discusiones de las diferencias culturales que marcan el género a partir de las diferencias sexuales, lo que al final ha desembocado en una discusión desde diversas perspectivas que lo han enriquecido a partir de sus desarrollos. Para Curiel (2007), se trata de un concepto bastante antiguo y suficientemente desarrollado a lo largo de cuatro siglos, el cual hace referencia a "un conjunto de características, rasgos, distinciones, funciones atribuidas a mujeres y hombres en las sociedades, a través de las cuales se construyen modelos" (Bonilla, 2010). Es por ello que el modo como se conciben las diferencias entre los sexos está íntimamente vinculado con aspectos culturales, sociales, económicos y políticos que se construyen en la interacción de los sujetos sociales. Pese a ello, es preciso diferenciar la lucha del desarrollo conceptual, pues la lucha es por alcanzar igualdad frente a las diferencias entre oportunidades y derechos, la categoría de género es una apuesta del siglo XX donde se ponen en discusión académica dichas desigualdades, que terminan sirviendo de soporte para algunas activistas feministas contemporáneas.

Frente a la categoría de género, Scott (1993) y otros autores lo asocian con una dimensión cultural, normativa, subjetiva y familiar, que inciden en cómo se concibe a la mujer y el hombre. En este sentido, se entiende como "una construcción social" (Comas, 1995) que trasciende y atraviesa todos los escenarios de la vida cotidiana. En este 
marco, las relaciones de poder ejercen un papel incipiente en la desigualdad de los sexos, y éstas se conciben a partir de estructuras de dominación que se fundamentan, según Bourdieu (2005), por entes institucionales como la familia, la iglesia, la escuela y el Estado; y actores masculinos que han promovido la subordinación de las mujeres.

Ahora bien, se hace una precisión acerca de las dinámicas de identidades de género en los grupos étnicos y sociales, los cuales se oponen con la diferenciación de roles que surgen en la población blanca, ya que para Bonilla (2010) los roles para estos grupos específicos se conciben sin ninguna distinción tanto para hombres como mujeres, porque se conciben como personas que tienen las capacidades físicas para ejercerlos, sin excluir alguna actividad económica para un sexo en particular. Lo que Wills define como regímenes de género, entendidos como "reglas de juego formales e informales que regulan las diferencias de género y que basadas en éstas distribuyen el poder”, lo cual permite profundizar en el análisis de la desigualdad y las violencias que han afectado y vulnerado los derechos de las mujeres. Al cruzar esto con las condiciones de etnia, clase, nivel educativo, religión, etc., se habla de intersección de género (Perilla, 2014). Este planteamiento ha sido debatido por Fraser (1997), quien considera las diferencias según el tipo de justicia al que se pueda acceder: de redistribución o reconocimiento; la primera hace referencia a la igualdad de los bienes y recursos sin alguna distinción; y la segunda, se asocia con el reconocimiento de la diferencia, específicamente a los grupos étnicos y de género.En este escenario, vale la pena retomar los planteamientos del feminismo latinoamericano, que sugiere la necesidad de retomar el reconocimiento de las experiencias localizadas, que especifiquen las condiciones de cada país, que permita incluso superar el "tráfico de teorías" (Femenías, 2007) y dar cuenta de mujeres como las que se retoman en el presente texto, mujeres campesinas, mujeres indígenas, mujeres afro, todas ellas con experiencias que enriquecen los desarrollos que se han establecido en este tema y que favorece "una conciencia de real contribución al discurso feminista".

En este sentido, un desarrollado visibilizado en mujeres campesinas es el empoderamiento, el cual vale la pena comprender desde la 
perspectiva teórica para una interpretación de las iniciativas ciudadanas con importante participación y liderazgo femenino y su significado como procesos articulados a formas de resistencia civil no violenta, lo cual se hizo desde tres acercamientos que resultan contrastantes y útiles para la caracterización de las experiencias identificadas con la Cartografía de la Esperanza. Estos tres acercamientos surgen de dar respuesta a las preguntas quiénes resisten, contra qué resisten o por qué resisten, pues es aparentemente en la conjunción de estos interrogantes que se identifican las mayores divergencias. Se tomó este camino consultando la tendencia actual del feminismo de la diferencia (Millán Benavides et al, 2004) que busca pluralizar el significado de ser hombre y mujer, de acuerdo con contextos históricos, culturales y locales específicos. El enfoque teórico de la resistencia no violenta se inicia con los contrastes de diversidad étnica y de pluralidad cultural, para dar paso a las discusiones identificadas en la literatura sobre género y lo que se conoce como aproximación neutra, desde las diferencias culturales y en las relaciones entre varones y mujeres.

En lo relacionado con paz, no todas las investigaciones presentan reflexiones conceptuales o teóricas sobre el tema de paz, pese a tenerlo como común denominador. El sustento teórico central en el tema de paz se remite a tres autores: Johan Galtung en lo relacionado con paz positiva y paz estructural; Vicenç Fisas frente al significado de la construcción de paz; Tafur (2011), a partir de los cuatro principios que componen la paz (natural, directa, estructural y cultural) remite fundamentalmente a los procesos de convivencia con resolución pacífica de conflictos en el marco de valores la solidaridad, respeto por sí mismo, bondad verbal (diálogo) y física (no agresión), equidad, libertad y solidaridad. El trabajo de De Ávila recupera el aporte de Fisas sobre el significado de los promotores de paz y afirma que una mujer constructora de paz "es quien interioriza e institucionaliza los valores, actitudes, conocimientos y procederes ligados a la cultura de la paz, desempeñando una función de alfabetización para la paz, ayudando a los otros y otras a aprender las dificultades de la mediación y de la transformación de los conflictos en cooperación” (2013:24). 
El anterior documento es un marco de referencia del rol que ocupa la mujer rural frente a escenarios de participación con miras a la construcción de paz, lo que trae consigo algunas dificultades que van desde los elementos históricos del papel de la mujer en espacios laborales y familiares, hasta el abordaje directo de la mujer en un territorio específico, como ocurre en Sumapaz.

\section{Referencias bibliográficas}

ARENAS, A.; COLLAZOS, C. “Colombia: Mujeres rurales gestionan su Ley”. Diálogos Textos breves sobre desarrollo rural solicitados por el IPDRS, 2010.

BONILLA, A. Trabajo doméstico y mujer rural: ...ésta vida mía. Bogotá, Universidad Nacional de Colombia. Facultad de Ciencias Humanas, 2010.

BOURDIEU, P. Capital cultural, escuela y espacio social. México, Siglo XXI, 2005. BUENDÍA-MARTÍNEZ, I. y CARRASCO, I. "Mujer, actividad emprendedora y desarrollo rural en América Latina y el Caribe”, en: Cuadernos de desarrollo rural, 10 (72), 2013. Pp. 21-45.

CASTRO, I. Y MOLINARI, C. "Ausencia de participación y marginación como forma de vida mujeres rurales en Chiapas (México)", en: Revista Clepsydra, 2014. Pp. 23-44.

COBO BEDIA, R. "Género", en: AA. VV. 10 palabras claves sobre mujer. Estella Navarra, Ed. Verbo Divino, 1995.

COMAS, D. “Trabajo, Género, Cultura”, en: Revista Complutense de Educación Norteamérica, 10, 1995. [En línea: 15/07/2017] Disponible en: http://revistas.ucm.es/ index.php/RCED/article/view/RCED9999220253B>.

DEERE, C., LEÓN, M. "Problemas metodológicos sobre la medición de la propiedad de la tierra por género". The Gender Asset Gap: Land in Latin America, 31, 2000. Pp. 925-947.

DEERE, C., LASTARRIA-CORNHIEL, S.; RANABOLDO, C. Tierra de mujeres. Reflexiones sobre el acceso de las mujeres rurales a la tierra en América Latina. La Paz, Bolivia: Fundación Tierra, 2011.

DE ÁVILA, M. "La Ruta Pacifica de las mujeres y su aporte en la construcción de paz en Colombia (Estudio de caso). Bogotá: Universidad Javeriana, 2013. [En línea: 23 de enero de 2017]. Disponible en: https://repository.javeriana.edu.co/handle/10554/15220

ESPINOSA, N; GIL, J; MESA, C. "La Mujer Rural Boyacense en una experiencia organizativa: descripción y análisis desde el Trabajo Social”, en: Revista Prospectiva, 17. Universidad del Valle, 2012. 
ESPINOSA, N.;.OYOLA, Y. "Mujeres rurales organizadas en torno al cultivo de la quinua”. Universidad Nacional de Colombia, 2012. Pp. 105-115.

FRASER, N. Justicia Interrupta. Reflexiones críticas desde la posición "postsocialista". Siglo del Hombre editores. Universidad de los Andes. Bogotá, 1997.

FEMENÍAS, M. "Esbozo de un feminismo latinoamericano", en: Estudios Feministas. Florianópolis. 15 (1):280, janeiro-abril 2007. Pp. 11-25.

FERRO, E. Perspectiva de género en la construcción de paz: análisis a partir de la Organización Femenina Popular y el marco integrado para la paz de John Paul Lederach. Bogotá: Universidad Javeriana, 2014. [En línea: 23 de Enero de 2017] Disponible en: https://repository.javeriana.edu.co/handle/10554/18597

IBARRA, M. "Acciones colectivas de las mujeres en contra de la guerra y por la paz en Colombia”, en: Revista Sociedad y Economía(13), 2007. Pp. 66-86. [En línea: 19 de Enero de 2017] Disponible en: http://www.redalyc.org/pdf/996/99616721004.pdf

JORDAN, F. "La economía campesina en la reactivación y el desarrollo agropecuario". Crisis Reactivación y Desarrollo. San Jose, IICA, 1989.

MARIN, I. "Resistencias desde la huerta. Movilización de mujeres en zonas rurales del suroccidente colombiano", en: La manzana de la discordia, 8(2), 2013. Pp. 89-107. [En línea: 15 de Enero de 2017] Disponible en: http://nexus.univalle.edu.co/index.php/ la_manzana_de_la_discordia/article/view/1542

MILLÁN DE BENAVIDES, C. "Estudios de familia y agendas emergentes”, en: Revista VIA IURIS Disponible en: <http://www.redalyc.org/articulo.oa?id=273931062007> ISSN 1909-5759

MZC (Mujeres en Zona de Conflicto); Corporación SISMA Mujer. "Mujeres en Zona de Conflicto. Diagnóstico: Mujer, paz y seguridad. Los movimientos de mujeres y paz en Colombia. Desde los años noventa hasta hoy”. Córdoba: Ayuntamiento de Córdoba, MZC, Corporación SISMA Mujer, 2010. [En línea: 23 de Enero de 2017] Disponible en: http://iknowpolitics.org/sites/default/files/diagnostico_colombia_definitivo_sep12.pdf

Parques Naturales de Colombia. (31 de julio de 2017). Parques Naturales de Colombia. Obtenido de http://www.parquesnacionales.gov.co/portal/es/parques-nacionales/ parque-nacional-natural-sumapaz/

PARRADO, S.; ISIDRO, L. "La paradoja hipócrita. Problematización de la participación política de la mujer misak”. Colombia Internacional, 2014. Pp. 135-170. Recuperado de http://www.redalyc.org/articulo.oa?id=81229999005

PÉREZ, J. Impacto del conflicto político militar en la vida cotidiana colombiana, la transformación de subjetividades y la construcción de sociedad civil: Localidades de Ciudad Bolívar y Sumapaz. 1991-2007. Bogotá: Universidad Javeriana, 2010. [En línea: 20 de Enero de 2017] Disponible en: https://repository.javeriana.edu.co/bitstream/ handle/10554/7736/tesis397.pdf? sequence $=1 \&$ isAllowed $=y$ 
Tensiones y retos sobre la mujer rural y su participación en la construcción de paz en Colombia

PERILLA, L. "Los roles de las mujeres rurales en el departamento de Nariño, Colombia. Tendencias y cambios”. Universidad Nacional de Colombia, 2014. Pp. 187-204.

PINTO, E. "Que cante la gallina, no solo el gallo: memoria, mujeres y tierra". Universidad Nacional de Colombia, 2011. Pp. 43-59.

Rosado, V. Impactos de los procesos políticos nacionales en las iniciativas organizativas de las mujeres piaroa en la Amazonia colombo-venezolana. Universidad Nacional de Colombia, 2011. Pp. 132-153.

SAÉNZ, M. Mujeres artesanas solicitantes de refugio en el Alto Apure venezolano una apuesta para la construcción de paz local desde estrategias comunitarias no violentas. Bogotá: Universidad Javeriana, 2013. [En línea: 21 de Enero de 2017] Dispobible en: https://repository.javeriana.edu.co/handle/10554/14259

SÁNCHEZ, M. L., \& MORÁ, M. I. "Paridad en la Representación Política de la Mujer Rural”, en: Revista de ciencias sociales aposta. 60, 2014. Pp. 1-38. [En línea: 15 de febrero de 2017] Disponible en: http://www.apostadigital.com/revistav3/hemeroteca/ msanoro1.pdf

SÁNCHEZ, M; RODRÍGUEZ, Z. "Acciones colectivas de las organizaciones de mujeres por la paz en Colombia”, en: Revista de Paz y Conflictos, 8(2), 2015. Pp. 149-177. [En línea: 19 de enero de 2017] Disponible en: http://revistaseug.ugr.es/index.php/revpaz/ article/viewFile/3190/3894.

SCOTT, J. "El género, una categoría útil para el análisis histórico”, en: NASH y AMELANG (eds.). Historia y género: las mujeres en la Europa moderna y contemporánea. Valencia, Alfons el Magnanim, 1990.

TAFUR RUEDA, M. "La construcción de paz desde el feminismo: una comparación de los movimientos de mujeres, la ruta pacífica y la red nacional de mujeres, sus discursos y prácticas en el escenario colombiano". Trabajo de Grado. Carrera de Ciencia Política. Bogotá, Pontificia Universidad Javeriana, 2011. [En línea: 20/02/2017] Disponible en: http://repository.javeriana.edu.co/bitstream/10554/7747/1/tesis434.pdf

SISMA. Corporación SISMA Mujer, Colombia. "Mujeres, Violencia Sexual en el Conflicto y el Proceso de Paz”. Bogotá, SISMA Mujer, 2014. [en línea: 20 de Enero de 2017] Disponible en: http://www.abcolombia.org.uk/downloads/Sexual_violence_ report_Spanish.pdf.

VARGAS, D. "Iniciativas Locales de Paz en Soacha". Estudio de caso de un proyecto de cambio social con enfoque de género. Bogotá, Pontificia Universidad Javeriana, 2014. [En línea: 18/01/2017] Disponible en: https://repository.javeriana.edu.co/handle/10554/13409

VILLARREAL, N. "Sectores campesinos, mujeres rurales y Estado en Colombia”. Tesis de doctorado. Universidad Autónoma de Barcelona. Facultad de Ciencias Políticas y Sociología. Programa de Estudios de Doctorado en Sociología. Barcelona, mayo de 2004.

VILLAREAL, N. Colombia: Violencias, conflicto armado y resistencias de género: las apuestas de una cartografía de la esperanza. Otras Miradas, 7(1), 2007. Pp. 50-66. [En línea: 12/01/2017] Disponible en: http://www.redalyc.org/html/183/18370105/ 
VILLARREAL, N. "Mujeres rurales y oportunidades económicas: de la participación al Empoderamiento”, en: Tendencias y Retos, Vol. 16. Bogotá, Universidad de la Salle, 2011.

ZULUAGA, G. "El Acceso a la Tierra Asunto Clave para las Mujeres Campesinas en Antioquia, Colombia”. Universidad Nacional de Colombia, 2011. Pp. 5949-5960.

ZULUAGA, G.; ARANGO, C. "Mujeres campesinas: resistencia, organización y agroecología en medio del conflicto armado", en: Cuadernos de Desarrollo Rural, 2013. Pp. 159-180. [En línea: 14/01/2017] Disponible en: http://revistas.javeriana.edu.co/index. $\mathrm{php} /$ desarrolloRural/article/view/7028/5587 